\title{
Validez y confiabilidad de la escala rasgo de metaconocimiento emocional (TMMS-24) en profesores universitarios 1
}

\section{Validity and reliability of the Trait Meta Mood Scale (TMMS -24) in University Teachers}

\author{
Rosalba Angulo Rincón ${ }^{2}$ \\ Ángela Pilar Albarracín Rodríguez ${ }^{3}$
}

\begin{abstract}
Resumen
Algunos estudios apoyan la concepción de que la inteligencia emocional (IE) constituye una variable predictora del desempeño laboral, siendo también útil en los procesos de selección del mejor candidato para las organizaciones. Es decir, personas con alto nivel de IE tienen una mayor probabilidad de ser exitosas en el campo profesional, al ser más adaptables a los acontecimientos vitales estresantes, empleando para ello estrategias de afrontamiento adecuadas, sumado a la capacidad que desarrollan para el trabajo en equipo. El objetivo de este estudio fue examinar la fiabilidad y validez de la escala rasgo de metaconocimiento emocional TMMS, en una muestra de docentes universitarios de instituciones privadas de educación superior, con el fin de aportar al conocimiento sobre su viabilidad en la evaluación de los niveles de inteligencia emocional inter e intrapersonal de esta población. En el estudio participaron 235 profesores universitarios con experiencia mínima de dos años, y vinculados a una institución de educación superior privada de la ciudad de Bucaramanga. El proceso comprendió la validación de contenido a través del criterio de jueces, prueba piloto, medición de la confiabilidad de la prueba por consistencia interna y la determinación de la validez de constructo, mediante el análisis factorial. Los resultados indican que la estructura factorial de la Escala TMMS-24 en la muestra evaluada está conformada por las tres dimensiones definidas en la versión española y que la escala reducida a 20 ítems constituye un instrumento con adecuados valores de validez y confiabilidad en la medición de la inteligencia emocional en profesores universitarios. En conclusión, se considera que esta escala constituye un instrumento útil para la medición e investigación del constructo inteligencia emocional como una variable organizacional importante para mejorar la calidad de vida laboral de los trabajadores y la dinámica organizacional de las instituciones de educación superior. Se sugiere hacer estudios en otras regiones del país con el fin de fortalecer la generalización de los resultados.
\end{abstract}

\section{Palabras clave}

Validación de pruebas, inteligencia emocional, profesores universitarios, organizaciones.

Códigos de clasificación JEL: C02, C16, C23

Artículo de investigación.

2 Universidad Pontificia Bolivariana, seccional Bucaramanga. Correo electrónico: rosalba.angulo@upb.edu.co. Código ORCID: 0000-0001-5341-8628

3 Universidad Pontificia Bolivariana, seccional Bucaramanga. Correo electrónico: angela.albarracín@upb.edu.co. Código ORCID: 0000-0002-9469-6613 


\begin{abstract}
Some studies support the conception of emotional intelligence - EI as a predictor of work performance, they are also useful in the selection process of the best candidate for organizations. That is, people with a high level of EI are more likely to be successful in the professional field, being more adaptable to stressful life events, using appropriate coping strategies, in addition to the ability they develop for teamwork. The objective of this study was to examine the reliability and validity of trait meta mood scale, in a sample of university professors from private institutions of higher education, in order to contribute to the knowledge about its viability in the evaluation of inter and intrapersonal emotional intelligence levels of this population. The study involved 235 university professors with a minimum experience of two years and linked to a private higher education institution in the city of Bucaramanga. The process included the validation of content through the criterion of judges, pilot test, measurement of the reliability of the test by internal consistency and the determination of construct validity, through factor analysis. The results indicate that the factorial structure of the TMMS-24 Scale in the evaluated sample is made up of the three dimensions defined in the Spanish version and that the scale reduced to 20 items constitutes an instrument with adequate values of validity and reliability in the measurement of Emotional intelligence in university professors. In conclusion, it is considered that this scale constitutes a useful instrument for measuring and researching the emotional intelligence construct as an important organizational variable to improve the quality of working life of workers and the organizational dynamics of higher education institutions. It is suggested to conduct studies in other regions of the country in order to strengthen the generalization of the results.
\end{abstract}

\title{
Keywords
}

Test validation, emotional intelligence, university professors, organizations.

\section{Introducción}

La inteligencia emocional (IE) como constructo multidimensional es definida como la habilidad para comprender, manejar las emociones propias y las de los demás, así como la posibilidad de regularlas y modificarlas (Bar-On, 2006; Salovey, Mayer, Goldman, Turvey \& Palfai, 1995). Durante las últimas décadas, la inteligencia emocional ha pasado de ser un concepto novedoso e inicialmente cuestionado, a constituirse en un elemento de especial interés para la comprensión de las diferencias individuales que pueden marcar la distinción entre las personas a la hora de alcanzar un buen desempeño académico o laboral, desarrollar competencias para la interacción y comunicación con los demás, acceder a un puesto de trabajo, emprender nuevos proyectos o a la posibilidad de predecir su comportamiento en contextos diversos. En particular, algunos autores sostienen que la investigación apoya la concepción de que la IE constituye una variable predictora del rendimiento de una persona en el ámbito laboral (Boyatzis \& Saatcioglu, 2008), lo que a su vez puede ser útil en los procesos de selección (García, García, \& Ramos, 2007). Es decir, personas con alto nivel de IE tienen una mayor probabilidad de ser exitosas en el campo profesional, al ser más adaptables a los acontecimientos vitales estresantes, empleando para ello estrategias de afrontamiento adecuadas, sumado a la capacidad que desarrollan para el trabajo en equipo (Bar-On, 1997; Nikolaou \& Tsaousis, 2002; Prati, Douglas, Ferris, Ammeter, \& Buckley, 2003; Weisinger, 1998).

El origen reciente de este constructo (IE) y el gran interés que ha suscitado en el ámbito profesional y científico durante las dos últimas décadas, ha promovido la creación de modelos teóricos para su explicación, de los cuales a su vez se derivan 
una variedad de instrumentos de medición. En la actualidad, dentro de los modelos mayormente aceptados se encuentran aquellos que describen la IE como un conjunto de competencias socioemocionales, habilidades cognitivas y rasgos estables de personalidad (mixtos). En este grupo se incluyen los modelos de Goleman (1995), BarOn (2006) y Boyatzis \& Saatcioglu (2008), que presentan un elemento en común, todos persiguen la capacidad de identificar y discriminar nuestras propias emociones y las de los demás, la capacidad de regular las emociones y de utilizarlas de forma adaptativa (Trujillo \& Rivas, 2005).

En particular, dentro de los modelos de Goleman (modelo de habilidad) se destaca el propuesto por Salovey \& Mayer (1990), fundamentado en el procesamiento de la información para comprender y regular las emociones. A partir de este, Salovey, Mayer, Goldman, Turvey \& Palfai (1995) diseñaron un instrumento para evaluar la inteligencia emocional intrapersonal percibida, más conocido como Trait Meta-Mood Scale (TMMS), conformado por 48 ítems agrupados en tres dimensiones: atención a las emociones $(\alpha=.86)$, claridad emocional $(\alpha=.87)$ y reparación emocional $(\alpha=.82)$. Posteriormente, los investigadores españoles Fernández, Extremera y Ramos (2004) obtuvieron una versión reducida de la TMMS, conformada por 24 ítems con una escala tipo Likert de 5 puntos que al igual que la original evalúa las dimensiones: atención a las emociones, claridad emocional y reparación emocional. La consistencia interna informada por los autores para cada una de las dimensiones fue: atención $(\alpha=.90)$, claridad $(\alpha=.90)$ y reparación $(\alpha=.86)$, consistencia interna ( $\alpha$ de Cronbach), presentando así mismo una adecuada fiabilidad test-retest.

Este instrumento ha sido ampliamente utilizado para evaluar la IE en los ámbitos laboral, educativo y familiar, tal como lo corroboran el meta-análisis realizado por Martins, Ramalho y Morin (2010) y el trabajo monográfico de Fernández, Berrios, Extremera y Augusto (2012) en el que señalan que de las pruebas de autoinforme empleadas durante los últimos años para su estudio, el 67\% corresponde a la TMMS. De esta tendencia dan cuenta algunos estudios en el contexto organizacional (Campo, Cervantes, Fontalvo, García \& Robles de la Cruz, 2008; Enríquez, Martínez \& Guevara, 2015) y educativo (Contreras, Barbosa \& Espinosa, 2010; Suárez, Guzmán, Medina \& Ceballos, 2012).

La investigación realizada por Campo et al. (2008) evaluó la inteligencia emocional percibida en Grumetes de la Escuela Naval de Suboficiales Armada República de Colombia en Barranquilla, evidenciando una correlación entre las tres dimensiones de la TMMS-24: percepción emocional, comprensión de sentimientos y regulación emocional. Además, los Grumetes que presentaron niveles altos de comprensión tenían la capacidad de reconocer las emociones en sí mismo y en la de los demás, así como de regularlas, promoviendo el crecimiento emocional e intelectual (Extremera \& Fernández, 2003; 2004). En conclusión, los Grumetes reflejaron puntajes buenos en la escala, lo que significa que la Inteligencia Emocional es un facilitador en la adaptación psicológica del individuo. 
Siguiendo la línea organizacional, encontramos el estudio realizado por Enríquez, Martínez \& Guevara (2015), cuyo objetivo fue determinar la relación entre inteligencia emocional y rendimiento laboral en una muestra de empleados de la organización Amanecer Médico, sede Cali. Los resultados mostraron una relación positiva entre la dimensión de comprensión de sentimientos y el desempeño laboral, lo que permite confirmar la importancia de la inteligencia emocional en la organización, al considerar la comprensión de los sentimientos y su regulación como un factor determinante en el desempeño laboral, favoreciendo el trabajo en equipo, la motivación para el logro de metas, la tolerancia a la frustración y el establecimiento de relaciones efectivas con los clientes. No obstante, a pesar de su amplia utilización, este instrumento aún no ha sido validado en Colombia, dando lugar a la presencia de sesgos de medición del constructo (IE) e incidiendo negativamente en la obtención de puntuaciones válidas, la toma de decisiones y en la calidad de las investigaciones. En la revisión de la literatura, se encuentra el estudio realizado por Espinoza, Sahueza, Ramírez \& Sáez (2015), quienes efectuaron la validación de constructo y confiabilidad de la TMMS-24 versión española, en estudiantes de enfermería de dos universidades de la comuna de Concepción en Chile, obteniendo coeficientes de Cronbach adecuados para las tres dimensiones (atención, $\alpha=.80$; claridad, $\alpha=79$ y regulación, $\alpha=85$ ), resultados similares a los registrados por los autores originales de la escala.

De esta manera, resulta de vital importancia disponer de instrumentos de medida validados en nuestro contexto que permitan evaluar en particular, las habilidades interpersonales e intrapersonales (IE) de los docentes universitarios, con el fin de contribuir no solo al desarrollo de la investigación sobre el tema, sino a la optimización de los procesos de selección de personal como la comprensión de los mecanismos de autoconocimiento y autorregulación de las emociones y su valor predictivo en las organizaciones. El objetivo del presente estudio consistió en examinar la validez y de la Escala de Inteligencia Emocional TMMS-24, versión española, en una muestra de docentes universitarios de instituciones privadas de educación superior de Bucaramanga y su área metropolitana.

\section{Método}

El presente estudio tiene un enfoque cuantitativo, de tipo instrumental y está orientado al abordaje de las propiedades psicométricas de las pruebas.

La muestra estuvo conformada por 235 docentes universitarios, de los cuales 102 son hombres $(43,40 \%)$ y 133 mujeres $(56,59 \%)$ con edades comprendidas entre 23 y 78 años $(M=40,4 ; D E=10,12)$, adscritos a 12 instituciones de educación superior privadas de la ciudad de Bucaramanga y su área metropolitana (63,15\% de la población total) las cuales fueron seleccionadas mediante un muestreo no probabilístico por conveniencia. Las instituciones participantes fueron: Universidad Autónoma de Bucaramanga (UNAB), Universidad Santo Tomás (USTA), Universidad Pontificia 
Bolivariana (UPB), Universidad de Santander (UDES), Universidad Antonio Nariño, Corporación Educativa de Colombia (Eforsalud), Tecnológica Fitec, Corporación Educativa ITAE, Corporación Universitaria de Investigación y Desarrollo (UDI), Corporación Universitaria Minuto de Dios (Uniminuto), Corporación Técnica de Colombia (Corpotec) y Congregación Mariana.

Los sujetos debían cumplir con los siguientes criterios de inclusión:

Ser docente universitario con experiencia mínima de dos años y estar vinculado a una universidad privada, independientemente del tipo de contrato establecido.

Para la selección de los participantes se estableció que cumplieran con los siguientes criterios de inclusión:

Ser docente universitario con experiencia mínima de dos años y estar vinculado a una universidad privada indiferentemente al tipo de contrato establecido.

De acuerdo con los criterios éticos dispuestos por la legislación colombiana, el estudio se enmarcó dentro de la investigación en seres humanos con riesgos mínimos, según el artículo 11, apartado B de la Resolución No. 008430 de 1993, de tal forma que todos los participantes fueron incluidos previo diligenciamiento del consentimiento informado, garantizando en todos los casos, el derecho a la confidencialidad y el anonimato.

Con relación en los instrumentos, se emplearon los siguientes:

- Ficha de Ingreso: Formato que registra los datos personales y clínicos de los participantes para corroborar los criterios de inclusión y exclusión de la investigación.

- Escala Rasgo de Metaconocimiento Emocional, TMMS-24: a partir del instrumento de medición de la inteligencia emocional, en idioma inglés, creado por Salovey, Mayer, Goldman, Turvey y Palfai (1995) para evaluar la inteligencia emocional percibida; la versión empleada en la presente investigación fue la adaptación realizada por Fernández, Extremera \& Ramos (2004) en población española, versión reducida y modificada que evalúa tres dimensiones: atención a los sentimientos, claridad emocional y regulación emocional, por medio de 24 ítems en una escala tipo Likert de cinco puntos (1 $=$ Totalmente en desacuerdo, $2=$ Algo de acuerdo, $3=$ Bastante de acuerdo, $4=$ Muy de acuerdo y $5=$ Totalmente de acuerdo. Con respecto a las propiedades psicométricas, muestra una fiabilidad alta (alpha de Cronbach) para cada dimensión (percepción, $\mathrm{a}=0.90$; claridad, $\mathrm{a}=0.90$; regulación $\mathrm{a}=0.86$ ) y una fiabilidad test-retest adecuada: percepción $=0.60$; comprensión $=0.70 \mathrm{y}$ regulación $=0.83$.

Para llevar a cabo el proceso de evaluación psicométrica de validez y confiabilidad de la Escala Rasgo de Metaconocimiento Emocional (TMMS-24), versión 
española, en primera instancia se procedió a la revisión de la validez de contenido, realizada mediante el juicio de 5 expertos del área de la psicología, quienes evaluaron el grado en que los ítems que conforman la escala, representan el contenido que el instrumento trata de medir. Luego, la TMMS-24 se aplicó a una prueba piloto de 30 docentes universitarios, evidenciando tanto la definición precisa del dominio como el juicio sobre el grado de suficiencia con que el dominio se evalúa (Leyva, 2011) con lo cual, no fue necesario efectuar modificaciones en la escala. Posteriormente, al contar con la autorización de las 12 universidades participantes, se procedió a reunir a los docentes de las diferentes áreas académicas, con el propósito de darles a conocer los objetivos, metodología del estudio y consultarles sobre su intención de participar en el mismo. Tras lo cual, los docentes participantes diligenciaron el consentimiento informado, la ficha de ingreso y aplicaron la prueba.

El tratamiento de los datos y los análisis estadísticos respectivos, se realizaron a través del paquete estadístico SPSS versión 24 (Statistical Package for the Social Sciences), empleando para ello análisis descriptivos y procedimientos de análisis más específicos para la obtención de pruebas de fiabilidad como el coeficiente Alfa de Cronbach y el programa FACTOR para análisis de validez de constructo por medio de análisis factoriales exploratorios (Martínez, 1995; Muñiz, 1996).

\section{Resultados}

En primer lugar, se presentarán los resultados del análisis factorial exploratorio de la Escala TMMS-24, seguidos de los resultados de confiabilidad.

Análisis factorial exploratorio (AFE): para este análisis se empleó el programa FACTOR. Por lo tanto, se inició con la extracción de la matriz Coeficiente de Pearson (correlación del producto-momento), la cual evidenció correlaciones moderadas alrededor de 0.30 entre los ítems de las tres dimensiones (percepción, comprensión y regulación), excepto los ítems 21, 22, 23 y 24, los cuales presentaron una correlación baja con los demás reactivos, por lo que es posible que estos ítems hagan referencia a un aspecto distinto del constructo evaluado. Igualmente, se calcularon las pruebas KMO (0.867) y esfericidad de Bartlett (chi cuadrado $=2459.4, \mathrm{p}<0.001)$, indicando viabilidad para realizar el análisis factorial de la escala (Lloret, Ferreres, Hernández \& Tomás, 2014).

Al realizar el análisis AFE por el método de Análisis Paralelo (AP) mostró que existen 3 factores comunes o dimensiones; de igual manera, al efectuar el análisis de componentes principales mostró que los ítems 21, 22, 23 y 24 no cargaron en ningún factor, por lo que se eliminaron al realizar un nuevo análisis. El segundo análisis mostró una estructura de tres dimensiones de la escala TMMS con autovalores por encima de 1.5 que explican el $60 \%$ de la varianza total (tabla 1). 
Validez y confiabilidad de la escala rasgo de metaconocimiento emocional...

Tabla 1. Varianza explicada de la Escala TMMS por los autovalores

\begin{tabular}{|c|c|c|c|}
\hline Ítem & Autovalores & Proporción varianza & $\begin{array}{c}\text { Proporción varianza } \\
\text { acumulada }\end{array}$ \\
\hline 1 & 5.82285 & 0.29114 & 0.29114 \\
\hline 2 & 4.42914 & 0.22146 & 0.5126 \\
\hline 3 & 1.76080 & 0.08804 & 0.60064 \\
\hline 4 & 1.03228 & 0.05161 & \\
\hline 5 & 0.83277 & 0.04164 & \\
\hline 6 & 0.74322 & 0.03716 & \\
\hline 7 & 0.66028 & 0.03301 & \\
\hline 8 & 0.56583 & 0.02829 & \\
\hline 9 & 0.51683 & 0.02584 & \\
\hline 10 & 0.50463 & 0.02523 & \\
\hline 11 & 0.43850 & 0.02192 & \\
\hline 12 & 0.40762 & 0.02038 & \\
\hline 13 & 0.35668 & 0.01783 & \\
\hline 14 & 0.34146 & 0.01707 & \\
\hline 15 & 0.32935 & 0.01647 & \\
\hline 16 & 0.30048 & 0.01502 & \\
\hline 17 & 0.29091 & 0.01455 & \\
\hline 18 & 0.24104 & 0.01205 & \\
\hline 19 & 0.22541 & 0.01127 & \\
\hline 20 & 0.19993 & 0.01000 & \\
\hline
\end{tabular}

Fuente. Elaboración propia.

Seguidamente, se obtuvo la matriz rotada donde se presenta cada dimensión con el valor de saturación de los ítems correspondientes mayores a 0.40 (tabla 2). De esta manera, en la primera dimensión o factor se agrupan los ítems del 1 al 8 que corresponden a la capacidad de sentir y expresar los sentimientos de forma adecuada; en la segunda dimensión se agrupan los ítems 9 a 16 que hacen referencia a la apropiada comprensión de los estados emocionales y en la tercera dimensión se encuentran los ítems 17 a 20, relacionados con la capacidad de regular los estados emocionales correctamente. Así mismo, los coeficientes de ajuste del modelo como el GFI (0.988), el RMSEA (0.023) y el AGFI (0.982) indican un adecuado ajuste de la estructura factorial de la escala. 
Tabla 2. Matriz factorial rotada de la Escala TMMS

\begin{tabular}{|c|c|c|c|}
\hline \multicolumn{4}{|c|}{ Matriz de carga rotada } \\
\hline \multirow{2}{*}{ Ítem } & \multicolumn{3}{|c|}{ Factor } \\
\hline & Percepción & Comprensión & Regulación \\
\hline 1. Presto mucha atención a los sentimientos. & 0,661 & & \\
\hline $\begin{array}{l}\text { 2. Normalmente me preocupo mucho por lo que } \\
\text { siento. }\end{array}$ & 0,772 & & \\
\hline $\begin{array}{l}\text { 3. Normalmente dedico tiempo a pensar en mis } \\
\text { emociones. }\end{array}$ & 0,835 & & \\
\hline $\begin{array}{l}\text { 4. Pienso que merece la pena prestar atención a } \\
\text { mis emociones y estado de ánimo. }\end{array}$ & 0,680 & & \\
\hline $\begin{array}{l}\text { 5. Dejo que mis sentimientos afecten a mis } \\
\text { pensamientos. }\end{array}$ & 0,720 & & \\
\hline 6. Pienso en mi estado de ánimo constantemente. & 0,776 & & \\
\hline 7. A menudo pienso en mis sentimientos. & 0,844 & & \\
\hline 8. Presto mucha atención a cómo me siento. & 0,684 & & \\
\hline 9. Tengo claros mis sentimientos. & & 0,630 & \\
\hline $\begin{array}{l}\text { 10. Frecuentemente puedo definir mis } \\
\text { sentimientos. }\end{array}$ & & 0,773 & \\
\hline 11. Casi siempre sé cómo me siento. & & 0,694 & \\
\hline $\begin{array}{l}\text { 12. Normalmente conozco mis sentimientos sobre } \\
\text { las personas. }\end{array}$ & & 0,609 & \\
\hline $\begin{array}{l}\text { 13. A menudo me doy cuenta de mis sentimientos } \\
\text { en diferentes situaciones. }\end{array}$ & & 0,728 & \\
\hline 14. Siempre puedo decir cómo me siento. & & 0,630 & \\
\hline 15. A veces puedo decir cuáles son mis emociones. & & 0,480 & \\
\hline 16. Puedo llegar a comprender mis sentimientos. & & 0,535 & \\
\hline $\begin{array}{l}\text { 17. Aunque a veces me siento triste, suelo tener } \\
\text { una visión optimista. }\end{array}$ & & & 0,749 \\
\hline $\begin{array}{l}\text { 18. Aunque me sienta mal, procuro pensar en cosas } \\
\text { agradables. }\end{array}$ & & & 0,781 \\
\hline $\begin{array}{l}\text { 19. Cuando estoy triste, pienso en todos los } \\
\text { placeres de la vida. }\end{array}$ & & & 0,720 \\
\hline $\begin{array}{l}\text { 20. Intento tener pensamientos positivos aunque } \\
\text { me sienta mal. }\end{array}$ & & & 0,831 \\
\hline Alfa de Cronbach & $\mathbf{0 , 8 8 3}$ & $\mathbf{0 , 8 8 9}$ & $\mathbf{0 , 8 5 5}$ \\
\hline
\end{tabular}

Fuente. Elaboración propia.

Análisis de consistencia interna: El análisis de consistencia interna empleado para la escala TMMS fue el coeficiente alfa de Cronbach, el cual evidenció valores entre 0.85 y 0.88 (tabla 2) para las diferentes dimensiones de la escala, mostrando una excelente consistencia y fiabilidad de la prueba en la medición de la inteligencia 
emocional en docentes universitarios, teniendo en cuenta la reducción de los ítems, en una versión de 20 ítems.

\section{Discusión}

El presente estudio tuvo como objetivo examinar la validez de la Escala de Inteligencia Emocional TMMS-24, versión española, en una muestra de docentes universitarios de instituciones privadas de educación superior de Bucaramanga y su área metropolitana.

De acuerdo con los resultados obtenidos se encontró que la estructura factorial de la Escala TMMS-24 en la muestra evaluada está conformada por tres dimensiones, siendo equivalente a la versión española de Fernández, Extremera y Ramos (2004); sin embargo, el número de ítems en la presente investigación tuvo que ser reducido por la falta de correlación y ajuste de la carga factorial según el análisis realizado.

En consecuencia, los ítems eliminados fueron el 21 "Si doy demasiadas vueltas a las cosas, complicándolas, trato de calmarme", el 22 "Me preocupo por tener un buen estado de ánimo", el 23 “Tengo mucha energía cuando me siento feliz” y el 24 "Cuando estoy enfadado intento cambiar mi estado de ánimo", que hacían parte de la dimensión Regulación, pero que al parecer en el contexto regional no muestran coherencia con la definición del constructo referido a la capacidad de regulación adecuada de los estados emocionales (Fernández, Extremera y Ramos, 2004).

De tal manera que, las tres dimensiones o factores resultantes del AFE quedaron conformadas por los siguientes ítems: la primera dimensión que agrupa los reactivos 1 al 8, y que son equivalentes a la versión española, se denomina Percepción, definida como la capacidad de la persona para sentir y expresar los sentimientos de forma apropiada (atención emocional). La segunda dimensión conformada por los reactivos 9 al 16 son compartidos con la versión de Fernández, Extremera y Ramos (2004) y se denomina Comprensión, referida a la claridad emocional, en el sentido de comprender adecuadamente los estados emocionales; y la tercera dimensión compuesta por los ítems 17 al 20, de los cuales los ítems 19 y 20 son iguales a los presentados por la versión española, mientras que el 17 y el 18 se agruparon de manera distinta en este factor, demostrando ser más coherentes con el constructo.

Por lo anterior, se evidencia que la escala mantiene su carácter multidimensional en la medición de la IE, siendo coherente con la definición propuesta por Bar-On (2006) y Salovey y Mayer et al. (1990). Igualmente, al analizar la confiabilidad de las tres dimensiones se encuentra una elevada consistencia interna en la evaluación de la IE. 


\section{Conclusiones}

Se evidencia que la Escala TMMS reducida a 20 ítems es un instrumento con adecuados valores de validez y confiabilidad en la medición de la IE en profesores universitarios de la región, el cual podría ser útil para un mayor avance de la investigación sobre el tema, en un contexto organizacional específico como las instituciones de educación superior. Específicamente, el estudio de la IE asociada a otros aspectos de la dinámica laboral que pueden contribuir a la solución de problemáticas que afectan el desempeño y calidad de vida laboral de esta población.

Finalmente, se recomienda la realización de estudios que empleen esta versión de la Escala validada en diferentes regiones del país, con el propósito de apoyar los resultados de esta investigación y propender por su generalización.

\section{Referencias}

Bar-On, R. (1997). Development of the Baron EQ-I: A measure of emotional and social intelligence. $105^{\text {th }}$ Annual Convention of the American Psychological Association in Chicago.

Bar-On, R. (2006). The Bar-On model of emotional-social intelligence (ESI). Psicothema, 18(supl), 13-25.

Bar-On, R., \& Parker, J. D. (2000). The emotional quotient inventorv: Youth version $(E Q-i: Y V)$. North Tonawanda, Nueva York: Multi-Health System.

Boyatzis, R.E., \& Saatcioglu, A. (2008). A review of trait meta-mood research. International Journal of Psychology Research, 2, 39-67.

Campo, L., Cervantes, G., Fontalvo, Z., García, M., \& Robles de la Cruz, G. (2008). Inteligencia emocional percibida en los grumetes de la Escuela Naval de Suboficiales A.R.C. Barranquilla. Psicogente, 11(20), 145-151.

Contreras, F., Barbosa, D., \& Espinosa, J. (2010). Personalidad, inteligencia emocional y afectividad en estudiantes universitarios de áreas empresariales implicaciones para la formación de líderes. Diversitas Perspectivas en Psicología, 6, 65-79.

Enríquez, E., Martínez, J., \& Guevara, L. (2015). Relación de la inteligencia emocional con el desempeño laboral. Ciencia \& Salud, 3(11), 41-46.

Espinoza, M., Sanhueza, O., Ramírez, N., \& Sáez, K. (2015). Validação do construto e da confiabilidade de uma escala de inteligência emocional aplicada a estudantes de enfermagem. Revista Latinoamericana Enfermagem, 23(1), 139-147, doi: https://doi.org/10.1590/0104-1169.3498.2535 
Extremera, N., \& Fernández, P. (2003). La inteligencia emocional en el contexto educativo: Hallazgos científicos de sus efectos en el aula. Revista de Educación, 332, 97- 116.

Extremera, N., \& Fernández, P. (2004). Inteligencia emocional, calidad de las relaciones interpersonales y empatía en estudiantes universitarios. Clínica y Salud, 15(2), 117-137.

Fernández, P., \& Extremera, N. (2008). A review of trait meta-mood research. International Journal of Psychology Research, 2, 39-67.

Fernández P., Extremera, N., \& Ramos, N. (2004). Validy and reliability of the Spanish modified version of the Trait Meta- Mood Scale. Psycological Reports, 94(3), 751-755.

Fernández, P., Berrios, P., Extremera, N. y Augusto, J. M. (2012). Inteligencia emocional: 22 años de avances empíricos. Behavioral Psychology, 20(1), 5-14.

García, A.L., García, M., \& Ramos, P.J. (2007). Aportaciones de la inteligencia emocional y la autoeficacia: aplicaciones para la selección de personal. Anales de Psicología, 23(2), 231-239.

Goleman, D. (1995). Emotional Intelligence. New York: Bantam Books.

Leyva, Y. E. (2011). Una reseña sobre la validez de constructo de pruebas referidas a criterio. Perfiles Educativos, 33(131), 131-154.

Lloret, S., Ferreres, A., Hernández, A., \& Tomás, I. (2014). El análisis factorial exploratorio de los ítems: Una guía práctica, revisada y actualizada. Anales de Psicología, 30(3), 1151-1169, doi: http://dx.doi.org/10.6018/analesps.30.3.199361

Mayer, J.D., \& Salovey, P. (2007). ¿Qué es inteligencia emocional? En Mestre, J.M. y Fernández, P. (Eds.). Manual de Inteligencia emocional, (pp. 25-45). Madrid: Pirámide.

Martínez, M.R. (1995). Psicometría: Teoría de los tests psicológicos y educativos. Madrid: Síntesis.

Martins, A., Ramalho, N., \& Morin, E. (2010). A comprehensive meta-analysis of the relationship between emotional intelligence and health. Personality and Individual Differences, 49(6), 554-564. doi: https://doi.org/10.1016/j.paid.2010.05.029

Muñiz, J. (1996). Teoría clásica de los test. Madrid: Ediciones Pirámide.

Nikolaou, I., \& Tsaousis, I. (2002). Emotional intelligence and occupational stress. The International Journal of Organizational Analysis, 10(4), 327-342.

Prati, L.; Douglas, C.; Ferris, G.; Ammeter, A., \& Buckley, M. (2003). Emotional intelligence, leadership effectiveness and team outcomes. The International Journal of Organizational Analysis, 11(1), 21-40. 
Salovey, P., \& Mayer, J. D. (1990). Emotional intelligence. Imagination, cognition and personality, 9(3), 185-211.

Salovey, P., Mayer, J. D., Goldman, S. L., Turvey, C., \& Palfai, T. P. (1995). Emotional attention, clarity, and repair: Exploring emotional intelligence using the Trait Meta-Mood Scale. En J. W. Pennebaker (Ed.). Emotion, Disclosure, \& Health, (pp. 125-151). Washington: American Psychological Association.

Suárez, Y., Guzmán, K., Medina, L., \& Ceballos, G. A. (2012). Características de inteligencia emocional y género en estudiantes de psicología y administración de empresas de una universidad pública de Santa Marta, Colombia: un estudio piloto. Duazary, 9(2), 132-139.

Thorndike, E.L. (1920). Intelligence and its uses. Harper's Magazine, 140, 227-235.

Trujillo, M., \& Rivas, L. (2005). Orígenes, evolución y modelos de inteligencia emocional. Innovar Revista de Ciencias Administrativas y Sociales, 15(25), 924-38.

Weisinger, H. (1998). Emotional Intelligence at work. San Francisco: Jossey-Bass. 